\title{
Enhanced Entrainment of Synchronous Inverters for Distributed Power Sources
}

\author{
Takashi HIKIHARA ${ }^{\dagger a)}$, Member, Tadashi SAWADA ${ }^{\dagger *}$, Nonmember, and Tsuyoshi FUNAKI ${ }^{\dagger b)}$, Member $^{\circ}$
}

\begin{abstract}
SUMMARY Synchronization has gained attention in science recently. In electrical engineering, a power network requires generators and loads to be in synchronization. The increase in distributed de power sources without sophisticated controls has made synchronization more difficult. This paper proposes a synchronous inverter designed for linking distributed power sources within a power network. The linkage inverters should have high confidential characteristics to keep the network stable. The frequency and phase synchronization between synchronous generators in power network has been acheived through power transmission systems. The synchronous inverters contribute in the development of the sophisticated power networks by providing distributed power sources that maintain synchronous operation.

key words: synchronization, distributed power source, power system, synchronous inverter, phase-locked loop
\end{abstract}

\section{Introduction}

A large number of systems that operate in synchronism have marked the advent of synchronization, which has gained attention in science recently [1]-[3] and also in engineering [4]. For more than 100 years, electrical engineers have worked to synchronize power [5] and communication networks. It denotes the development of electric and electronic engineering.

An electric power network is a huge system that couples synchronous generators throughout the power transmission network. Over the last few decades, synchronus systems have become common and highly reliable. Recently, the competition in wholesale electricity has increased the stress on the power delivery system. Moreover, small generators and storage devices (distributed power sources) are distributed throughout the networks, and are seamlessly integrated into the power delivery system [6]. The increase of distributed power sources requires a method for their flexible interconnection to power delivery systems [7].

Dc distributed power sources, for example, solar cells, fuel cells, and secondary batteries, connect to ac power delivery system through inverters. The inverters operate synchronously in the network. However, conventional inverters have no intrinsic advantage to synchronize their output with an ac power system. The controller decides the frequency

Manuscript received January 9, 2007.

Manuscript revised June 21, 2007.

Final manuscript received August 8, 2007.

${ }^{\dagger}$ The authors are with the Department of Electrical Engineering, Kyoto University, Kyoto-shi, 615-8510 Japan.

*Presently, with JR Central.

a) E-mail: hikihara@kuee.kyoto-u.ac.jp

b)E-mail: funaki@kuee.kyoto-u.ac.jp

DOI: 10.1093/ietfec/e90-a.11.2516 and phase of the inverter's control signal for pulse width modulation on the basis of power system [8]-[12]. Thus, the synchronization is one-directionally determined by the triggering signal.

Generally, in distributed power systems, the power factor of inverter is kept at 1.0 without considering the state of the power system [9]. The regulator extracts the maximum active power through the inverters. On the other hand, synchronous generators autonomously adjust themselves to the power system depending on the load and the mechanical input which implies the bidirectional synchronization through power. Therefore, it is beneficial to provide this characteristic in inverters under load.

This paper is organized as follows. A voltage controlled inverter (VCI), proposed by Harada et al. [13], is examined. Their proposed VCI scheme tracks the maximum power point while maintaining frequency synchronization. Here we additionally confirm that VCI first achieves the frequency synchronization and an additionally attached phaselocked loop (PLL) later acheives the phase entrainment. The VCI and PLL configuration possesses the ability of a frequency and phase synchronization depending on the state of distributed power source and power network. The improved inverter is called a synchronous inverter (SI). The steady state advantage is also kept in the newly proposed inverters in this paper. Root analysis confirms that the SI achieves stability at frequency and phase synchronism. Furthermore, the characteristics of VCI and SI are numerically and experimentally discussed. Finally, the frequency and phase synchronization is discussed from the viewpoint of global dynamics. The entrainment behaviors caused by disturbances are numerically discussed using projected phaseplane approach for state space by estimating the domain of attraction.

The concept of SI is inspired by characteristics of synchronous generators, which dynamics is described by a swing equation. The transient behavior and dynamics of power networks are substantially described by coupled swing oscillators with transmission lines. The distributed power sources keep the network synchronized by adopting the proposed SI and act cooperatively within the organization of a highly sophisticated power delivery system. 


\section{Principles of Synchronous Inverter}

\subsection{Configuration of Distributed Power System}

A distributed power system and its configuration are explained in this section. Figure 1 shows the typical configuration of a single-phase distributed power system. The inverter, named INV in the figure, converts the dc voltage $E_{\mathrm{I}}$ to ac voltage $e_{\text {inv }}$. The distributed power source is assumed as a dc voltage source $E_{s}$ with an internal resistor $R_{s}$. In the solar cells, $E_{\mathrm{s}}$ changes abruptly according to the weather and shadows of the cloud. $C$ plays a role to stabilize the change of $E_{\mathrm{I}}$, which is larger than the conventional smoothing capacitor.

\subsection{Voltage Controlled Inverter (VCI)}

In 1986, Harada et al. proposed a VCI for interfacing inverters to power systems [13]. It did not track but followed the maximum power point of the solar cell while keeping the inverter frequency synchronous with the power network. Here, we focus on the characteristics of synchronization with keeping the ability of maximum power point tracking in steady state. Figure 2 shows the block diagram of the VCI that produces a PWM reference signal. Further, we describe VCI from the viewpoint of synchronization.

The VCI determines a PWM reference signal with an angular frequency $\omega$ by regulating $E_{\mathrm{I}}$. Assume that the synchronous angular frequency $\omega_{0}$ is given by the voltage $E_{\mathrm{OPT}}$. Here the suffix "OPT" implies the optimal value for $\omega_{0}$. Once the reference signal is given, the inverter circuit acts as a power amplifier with a constant voltage.

The principle of VCI is explained as follows (see Fig. 2). Here, the angular frequency $\omega_{\text {inv }}$ for the PWM reference signal is given by

$$
\omega_{\text {inv }}=K E_{\mathrm{I}},
$$

where $K$ is the linear gain. When we depict the difference of angular frequencies between $\omega_{\text {inv }}$ and $\omega_{0}$ by $\Delta \omega=\omega_{\text {inv }}-\omega_{0}$,

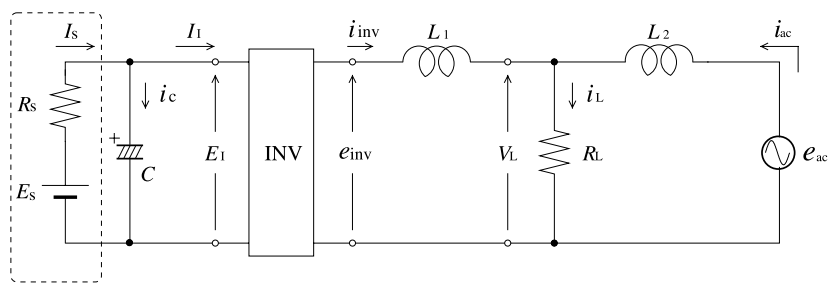

Fig. 1 Configuration of distributed power system and linkage inverter to power delivery system.

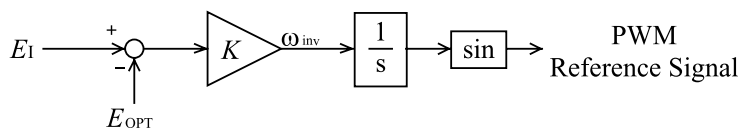

Fig. 2 Block diagram of VCI. the following relation is obtained:

$$
\frac{\mathrm{d} \Delta \omega}{\mathrm{d} t}=K \frac{\mathrm{d} E_{\mathrm{I}}}{\mathrm{d} t}
$$

if $\omega_{0}$ is constant. The current of de filtering capacitance $i_{\mathrm{C}}$ in Fig. 1 is represented by

$$
i_{\mathrm{C}}=C \frac{\mathrm{d} E_{\mathrm{I}}}{\mathrm{d} t}=\frac{E_{\mathrm{s}}-E_{\mathrm{I}}}{R_{\mathrm{s}}}-I_{\mathrm{I}},
$$

where $i_{\mathrm{C}}$ is the averaged value in a period. From Eqs. (1), (2), and (3), the following differential equation is obtained:

$$
\left\{\begin{array}{l}
\frac{\mathrm{d} \delta}{\mathrm{d} t}=\Delta \omega \\
\frac{\mathrm{d} \Delta \omega}{\mathrm{d} t}=\frac{K}{C}\left(\frac{E_{\mathrm{s}}-E_{\mathrm{OPT}}}{R_{\mathrm{S}}}-I_{\mathrm{I}}\right)-\frac{1}{C R_{\mathrm{S}}} \Delta \omega,
\end{array}\right.
$$

where $\delta$ corresponds to the phase difference between the fundamental components of inverter voltage $e_{\text {inv }}$ and the voltage $V_{\mathrm{L}}$ at the point of common coupling. The equation represents the VCI dynamics, and has the ability to adjust the frequency of the inverter's output voltage at $\omega_{0}$.

The optimal angular frequency $\omega_{0}$ is assumed at $E_{\mathrm{I}}=$ $E_{\mathrm{OPT}}$. If dc voltage $E_{\mathrm{I}}$ becomes higher than $E_{\mathrm{OPT}}$ and $\omega_{\text {inv }}$ increases from the angular frequency $\omega_{0}$, then the phase angle difference $\delta$ increases. This increases the output current $I_{\text {inv }}$ and the source current $I_{\mathrm{I}}$. As a result, the dc voltage $E_{\mathrm{I}}$ decreases. On the other hand, if $E_{\mathrm{I}}$ becomes lower than $E_{\mathrm{OPT}}$, then $E_{\mathrm{I}}$ increases. Therefore, the angular frequency $\omega_{\text {inv }}$ is kept at $\omega_{0}$, and the frequency synchronization is acheived. However, the characteristic is strongly governed by circuit parameters, $R_{s}$ and $C$. This approach does not consider the phase synchronism. Furthermore, the VCI proposed by Harada et al. had additional regulation to follow the maximum power point keeping the power factor at 1.0. Therefore, the phase synchronization is established under the control to keep the equilibrium point.

\subsection{Synchronous Inverter}

In this section, we propose a synchronous inverter (SI) based on VCI. Even after VCI establishes the frequency synchronization, it is difficult to link the distributed power source to power delivery systems because of the phase difference. At the onset of regulation of the reference frequency, the phase differences increase the power flow between distributed power source and the power system. When the phase appears delayed, the power inflow decreases $I_{I}$ and increases $E_{\mathrm{I}}$. Thus, the frequency increases until the phase difference coincides with zero. If the phase difference is leading the power system phase, then the frequency is reduced. As a result, VCI autonomously establishes the frequency synchronization. However, the frequency and phase synchronization is not ensured under disturbances.

The development of SI aims to resolve the difficulties of the phase synchronization under disturbances, while maintaining frequency synchronization. The SI adds a PLL 
to adjust the output phase to the linkage point. This inverter is called SI-I and the block diagram is shown in Fig. 3. The additional angular frequency $\omega_{\text {pll }}$ is given by PLL. The PLL tracks the variations in the voltage at the linkage point. Thus, around the steady state $\omega_{\text {pll }}$ corresponds to $E_{\mathrm{OPT}}$.

Figure 4 shows the block diagram of an other inverter controller, referred to as SI-II in this paper. The controller is designed to improve the transient characteristics for synchronization. It adopts the differential signal $\Delta \omega_{\text {pll }}$, which is the state variable in PLL, directly into the original control block to regulate the angular frequency. In SI-II, if $\omega_{\text {pll }}$ does not have direct relation with $E_{\mathrm{OPT}}$, then an error remains. Therefore we select the differential signal in PLL to avoid the errors in the setting of $E_{\mathrm{OPT}}$ for $\omega_{0}$. In Fig. $4, \omega_{0}^{*}$ should be ideally given by $E_{\mathrm{OPT}}$. If $\omega_{0}^{*}=\omega_{0}$, then SI-II becomes same as SI-I. However, if the inductance $L_{2}$ cannot be neglected in the transmission line, SI-II has a possibility to generate the lead and lag voltage by changing the sign of $\Delta \omega_{\text {pll }}$ added to $\Delta \omega_{\text {avr }}$. In this paper, only the lead voltage is considered.

\subsection{Mathematical Formulation of Linkage Methods}

\subsubsection{SI-I}

The controller shown in Fig. 3 assumes that the low pass filter, which is depicted as LPF in Fig. 3, is ideal and the harmonic components are completely eliminated in PLL. The derivative of angular frequency $\Delta \omega_{\text {pll }}$ is ideally depicted by

$$
\Delta \omega_{\mathrm{pll}}=-K_{\mathrm{p}} \sin \left(\delta_{\mathrm{pll}}-\delta_{\mathrm{ref}}\right),
$$

where $\delta_{\text {pll }}$ and $\delta_{\text {ref }}$ are the phase angles of the sinusoidal out-

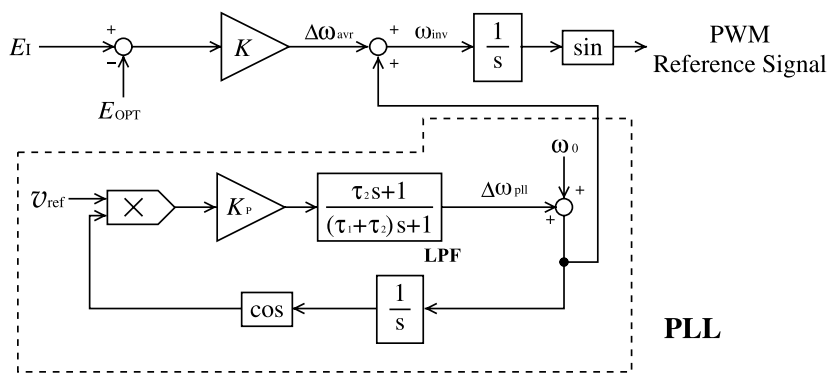

Fig. 3 Block diagram of SI-I.

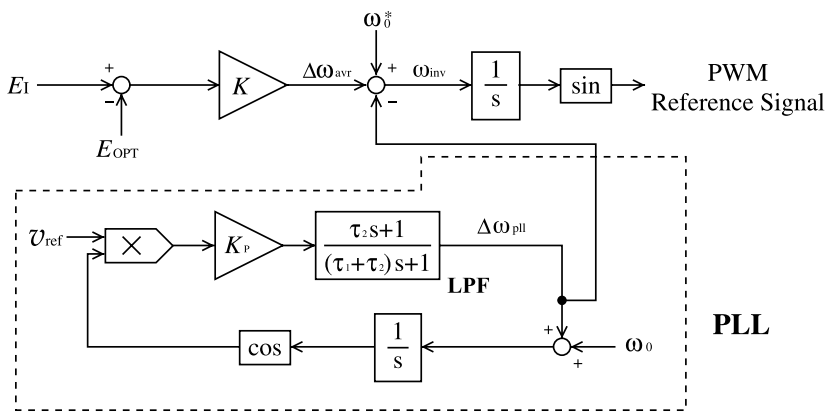

Fig. 4 Block diagram of SI-II. put voltage of the PLL and the voltage at the linkage point, respectively. $\delta_{\text {ref }}$ is given by reference sinusoidal input $v_{\text {ref }}$. The angular frequency deviation $\Delta \omega_{\mathrm{avr}}$ of the dc automatic voltage regulator (dcavr) is represented by

$$
\Delta \omega_{\mathrm{avr}}=K\left(E_{\mathrm{I}}-E_{\mathrm{OPT}}\right) .
$$

From Eqs. (5) and (6), the improved system equation is formulated as follows:

$$
\left\{\begin{aligned}
\frac{\mathrm{d} \delta_{\mathrm{avr}}}{\mathrm{d} t} & =\Delta \omega_{\mathrm{avr}} \\
\frac{\mathrm{d} \Delta \omega_{\mathrm{avr}}}{\mathrm{d} t} & =\frac{K}{C}\left(\frac{E_{\mathrm{s}}-E_{\mathrm{OPT}}}{R_{\mathrm{s}}}-I_{\mathrm{I}}\right)-\frac{1}{C R_{\mathrm{s}}} \Delta \omega_{\mathrm{avr}} \\
\frac{\mathrm{d} \delta_{\mathrm{pll}}}{\mathrm{d} t} & =\Delta \omega_{\mathrm{pll}}=-K_{\mathrm{p}} \sin \left(\delta_{\mathrm{pll}}-\delta_{\mathrm{ref}}\right) \\
\delta & =\delta_{\mathrm{avr}}+\delta_{\mathrm{pll}} \\
\Delta \omega & =\Delta \omega_{\mathrm{avr}}+\Delta \omega_{\mathrm{pll}} .
\end{aligned}\right.
$$

Where $\delta_{\text {avr }}$ denotes the phase angle difference based on $\Delta \omega_{\mathrm{avr}}$. The dynamics of synchronization is correctly described by Eq. (7).

\subsubsection{SI-II}

Just as with the SI-I, LPF in the PLL is assumed to be ideal. Thus, the system equation to generate the PWM reference signal for the inverter is formulated as follows:

$$
\left\{\begin{aligned}
\frac{\mathrm{d} \delta_{\mathrm{avr}}}{\mathrm{d} t} & =\Delta \omega_{\mathrm{avr}} \\
\frac{\mathrm{d} \Delta \omega_{\mathrm{avr}}}{\mathrm{d} t} & =\frac{K}{C}\left(\frac{E_{\mathrm{s}}-E_{\mathrm{OPT}}}{R_{\mathrm{s}}}-I_{\mathrm{I}}\right)-\frac{1}{C R_{\mathrm{s}}} \Delta \omega_{\mathrm{avr}} \\
\frac{\mathrm{d} \delta_{\mathrm{pll}}}{\mathrm{d} t} & =-\Delta \omega_{\mathrm{pll}}=-K_{\mathrm{p}} \sin \left(\delta_{\mathrm{pll}}+\delta_{\mathrm{ref}}\right) \\
\delta & =\delta_{\mathrm{avr}}+\delta_{\mathrm{pll}} \\
\Delta \omega & =\Delta \omega_{\mathrm{avr}}+\Delta \omega_{\mathrm{pll}} .
\end{aligned}\right.
$$

Here, we set $\omega_{0}^{*}=\omega_{0}$. Equation (8) is not remarkably different from Eq. (7), except for the sign in the third equation. However, the sign makes it possible to feed lead voltage, which compensates the effect by inductances in the transmission line. Thus, this possibility seems an important practical difference. Furthermore, there is a room to apply a control loop to adjust the power factor.

\subsection{Operation of Inverter and PLL}

In this section, the characteristics of inverters are discussed based on the phasor diagram in ac circuit operation. The time constants in dynamics described by Eqs. (4), (7), and (8) are relatively larger than the period of ac voltage. Therefore, the relationships of the fundamental components govern the dynamics of the inverters. This discussion helps us to understand the electrical phase relation of additional PLL for synchronous inverters to the phase of distributed power 
line. Even in the case of transient state, in which each phase varies depending on time, the phase relation of fundamental components are almost kept. The phase is referred by PLL in each the control method. This is the reason that the discussion based on the phasor diagram is included here.

\subsubsection{Connection through $L_{1}$}

First, we consider that the inverter is connected to power delivery system without linkage inductance $L_{2}$. That is, $L_{2}=0$. In ac electrical circuit analysis, the fundamental components are considered by phasor. The time-dependent component $e^{j \omega_{0} t}$ is abbreviated in the expressions. This description is relevant to the time constant towards synchronization. The following discussion neglects the higher harmonic components in the electrical circuit. The fundamental components are described by complex variables according to their amplitudes and phases with the expression (). They are called phasor. The variables without $\hat{()}$ correspond to the absolute value.

If linkage inductance $L_{2}$ between power delivery system and a load is set at 0 , then the phase angle difference is determined by referring ac voltage at the point of common coupling. The phasor diagram for the circuit in Fig. 1 is shown in Fig. 5. Here, we assume $\left|\hat{E}_{\text {inv }}\right|=\left|\hat{E}_{\text {ac }}\right|=E$. It does not loose generality. Figure 5 gives the following relation:

$$
2 E \sin \frac{\delta}{2}=\left|j X \hat{I}_{\text {inv }}\right|,
$$

where $X=\omega_{0} L_{1}$. If we neglect the loss in inverter, then the power preserving characteristic is kept between input dc power $E_{\mathrm{I}} I_{\mathrm{I}}$ and output ac power $E_{\mathrm{inv}} I_{\mathrm{inv}}$, which consists of the dc component depending on the phase angle difference $\delta / 2$ between them and ac component with double $\omega_{0}$. The former component corresponds to the active electric power and the latter to reactive electric power based on the fundamental components. Assuming that $E_{\mathrm{I}}$ is constant at $E, I_{\mathrm{I}}$ is represented by

$$
I_{\mathrm{I}}=\frac{E}{X} \sin \frac{\delta}{2}\left\{\cos \frac{\delta}{2}-\cos \left(2 \omega t+\frac{3}{2} \delta\right)\right\} .
$$

The second term in Eq. (10) can be eliminated by dc capacitance $C$. Then $I_{\mathrm{I}}$ is given by

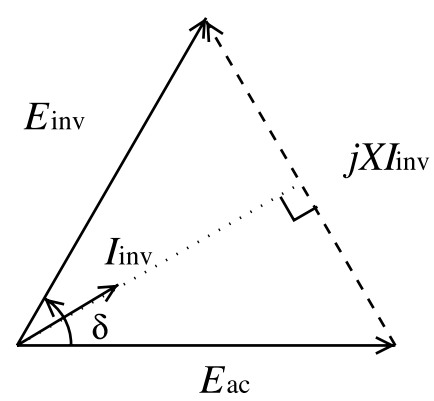

Fig. 5 Phasor diagram of linkage circuit with $L_{2}=0$.

$$
I_{\mathrm{I}}=\frac{E}{X} \sin \frac{\delta}{2} \cos \frac{\delta}{2}=\frac{E}{2 X} \sin \delta
$$

The phase angle difference $\delta_{\text {ref }}$ in Eq. (7) between the PLL and power system equals to zero because the PLL reference voltage $V_{\mathrm{L}}$ is directly determined by $E_{\mathrm{ac}}$. Thus, the reactive electric power in the ac side is absorbed by the capacitor in the dc side. Therefore, the third term of Eq. (7) is represented by

$$
\frac{\mathrm{d} \delta_{\mathrm{pll}}}{\mathrm{d} t}=-K_{\mathrm{p}} \sin \delta_{\mathrm{pll}}
$$

\subsubsection{Connection through $L_{1}$ and $L_{2}$}

Here, we consider that the inductance $L_{2}$ can not be neglected in the transmission line. The output current of the inverter $\hat{I}_{\text {inv }}$ and the current from power system $\hat{I}_{\mathrm{ac}}$ are represented by

$$
\left\{\begin{array}{c}
\hat{I}_{\text {inv }}=\frac{\hat{E}_{\mathrm{inv}}-\hat{V}_{\mathrm{L}}}{j X_{1}}, \\
\hat{I}_{\mathrm{ac}}=\frac{\hat{E}_{\mathrm{ac}}-\hat{V}_{\mathrm{L}}}{j X_{2}}, \\
\hat{I}_{\mathrm{ac}}+\hat{I}_{\mathrm{inv}}=\hat{I}_{\mathrm{L}}=\frac{\hat{V}_{\mathrm{L}}}{R_{\mathrm{L}}},
\end{array}\right.
$$

where $X_{1}=\omega_{0} L_{1}$ and $X_{2}=\omega_{0} L_{2}$. By Eq. (13), $I_{\text {inv }}$ is obtained as follows:

$$
\hat{I}_{\mathrm{inv}}=\frac{R_{\mathrm{L}}}{j Z}\left\{\left(1+j \frac{X_{2}}{R_{\mathrm{L}}}\right) \hat{E}_{\mathrm{inv}}-\hat{E}_{\mathrm{ac}}\right\},
$$

where $Z=\omega_{0} L_{1} R_{\mathrm{L}}+\omega_{0} L_{2} R_{\mathrm{L}}+j \omega_{0}^{2} L_{1} L_{2}$.

Here, we set a condition $X_{1}=X_{2}=X$ and $X=$ $\alpha R_{\mathrm{L}}(\alpha \neq 0)$ for simplicity. As a result, $\hat{I}_{\text {inv }}$ is given by

$$
\hat{I}_{\mathrm{inv}}=\frac{1}{j X\{2+j \alpha\}}\left\{(1+j \alpha) \hat{E}_{\mathrm{inv}}-\hat{E}_{\mathrm{ac}}\right\} .
$$

The phasor diagram is described in Fig. 6 based on Eq. (15), where the angles $\theta$ and $\sigma$ are constant with relation to circuit parameters.

The absolute value $I_{\text {inv }}$ is expressed as

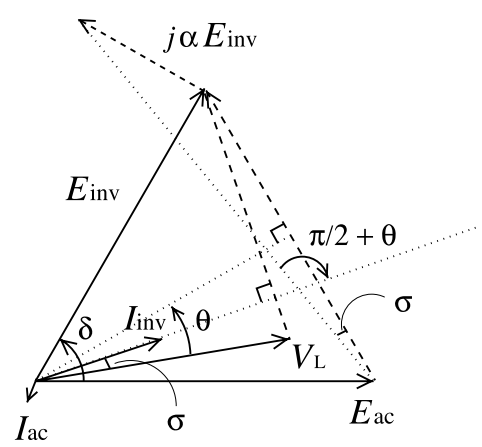

Fig. 6 Phasor diagram of linkage circuit with $L_{2}=L_{1}$. 


$$
I_{\mathrm{inv}}=\frac{\alpha E \sin \frac{\delta}{2}}{\sin \sigma} \cdot \frac{1}{X} \cdot \frac{1}{\sqrt{4+\alpha^{2}}} \cdot \sin \left(\omega t+\frac{\delta}{2}-\theta+\sigma\right) .
$$

Thus, $I_{\mathrm{I}}$ is given by

$$
I_{\mathrm{I}}=\frac{E}{2 X} \cdot \frac{1}{4+\alpha^{2}}(2 \sin \delta+\alpha \cos \delta+\alpha) .
$$

Figure 6 shows $\delta_{\text {ref }}$ equals to $\delta / 2-\theta=\left(\delta_{\text {avr }}+\delta_{\text {pll }}\right) / 2-\theta$. This implies that the third equation in Eq. (7) should be given by

$$
\frac{\mathrm{d} \delta_{\mathrm{pll}}}{\mathrm{d} t}=-K_{\mathrm{p}} \sin \left\{\frac{1}{2}\left(\delta_{\mathrm{pll}}-\delta_{\mathrm{avr}}\right)+\theta\right\} .
$$

This section presented models of proposed synchronous inverters with PLL. They are based on the operation of PLL and relations of fundamental components in electric circuit. The self-synchronizing dynamics of the inverters are restricted in the phase by the power relations determined by their electric circuit.

\section{Numerically Simulated Performance of Inverters}

In this section, the performances of the linkage inverters are numerically discussed. The circuit and control parameters are given in Table 1.

From the practical viewpoint, there are two types of disturbances that occur during linkage operations: (i) a sudden phase shift of ac voltage from 0 to $0.3 \pi \mathrm{rad}(\mathrm{Lag})$ and (ii) a sudden decrease of dc source voltage $E_{\mathrm{s}}$ from $60 \mathrm{~V}$ to $52 \mathrm{~V}$. In the following discussions the origin of temporal axis is at the onset of disturbance. The numerical simulations were performed by MATLAB6.1 and the results were confirmed by proprietary C-language program code.

\subsection{Connection through $L_{2}$}

In the connection through $L_{1}$, the transient response by SI-I is numerically compared with the response by VCI. Here, the gain $K_{\mathrm{p}}$ in the PLL is set at 10 . First, we estimate the phase difference $\delta$ between the voltage $E_{\text {inv }}$ of the linkage inverter and the voltage $V_{\mathrm{L}}$ at the linkage node. When a sudden phase shift is applied to $V_{\mathrm{L}}$ at $0.3 \pi(\mathrm{Lag})$, the inverters regulate the phase of $E_{\text {inv }}$ to $V_{\mathrm{L}}$, while maintaining the synchronization.

Figure 7 represents the response to transients by VCI and SI-I. The SI-I shows an improved response. In Eqs. (4) and (7), the dc capacitance $C$ has a role to response as an inertia of dynamics. Figure 8 shows the effect of capacitance on transient response. The natural frequency of the swing decreases as the capacitance increases. However, the swing remains in large capacitance.

On the other hand, $\delta$ does not show any dependency on the sudden drop of dc source voltage, as shown in Fig. 9. The transient characteristics of SI-I completely coincide with the result by VCI. This illustrates that the PLL does not govern the transient characteristics caused by disturbances in the distributed power source.
Table 1 Circuit and control settings.

\begin{tabular}{|c|c|}
\hline$E_{s}$ & $60 \mathrm{~V}$ \\
\hline$R_{s}$ & $20 \Omega$ \\
\hline$C$ & $60 \mathrm{mF}$ \\
\hline$L_{1}$ & $60 \mathrm{mH}$ \\
\hline$e_{\mathrm{AC}}$ & $50 \sin \omega_{0} t \mathrm{~V}$ \\
\hline$\omega_{0}$ & $120 \pi \mathrm{rad} / \mathrm{s}$ \\
\hline$K$ & $2.4 \pi$ \\
\hline
\end{tabular}

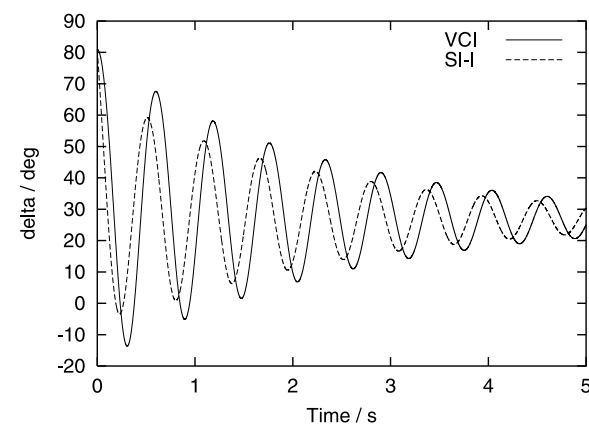

Fig. 7 Transient response caused by sudden phase shift of ac voltage of linkage node with $L_{2}=0$ from 0 to $0.3 \pi$ at $0 \mathrm{~s}$.

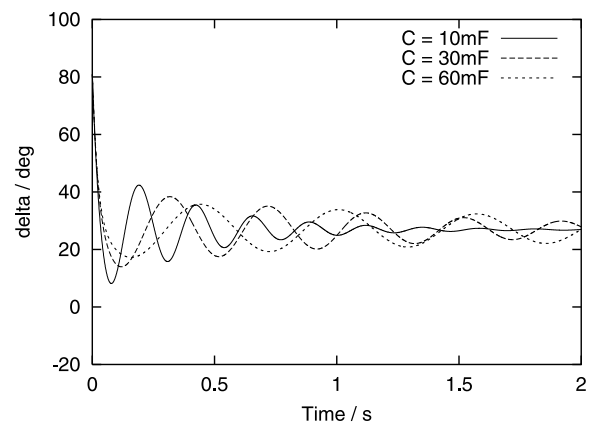

Fig. 8 Dependency of transient response of SI-I on capacitance at sudden phase shift $C$ while keeping $L_{2}=0$.

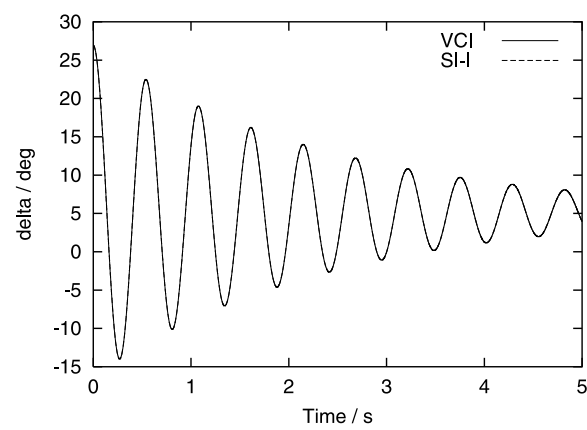

Fig. 9 Transeinet response caused by sudden drop of dc source voltage $E_{\mathrm{s}}$ from $60 \mathrm{~V}$ to $52 \mathrm{~V}$ under $L_{2}=0$.

\subsection{Connection through $L_{1}$ and $L_{2}$}

Here, we discuss the case in which the inductance $L_{2}$ cannot be neglected and compare SI-II with VCI. In the simulation, $L_{2}$ is set at $60 \mathrm{mH}$ that equals to $L_{1}$. The gain $K_{\mathrm{p}}$ in PLL 


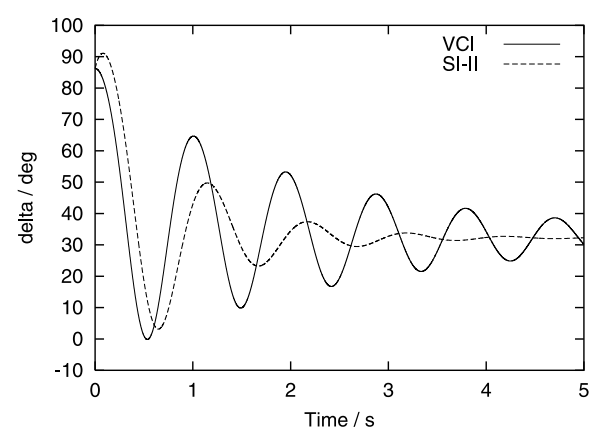

Fig. 10 Transient response caused by sudden phase shift of ac voltage at linkage node with $L_{1}=L_{2}$ from 0 to $0.3 \pi(\mathrm{Lag})$.

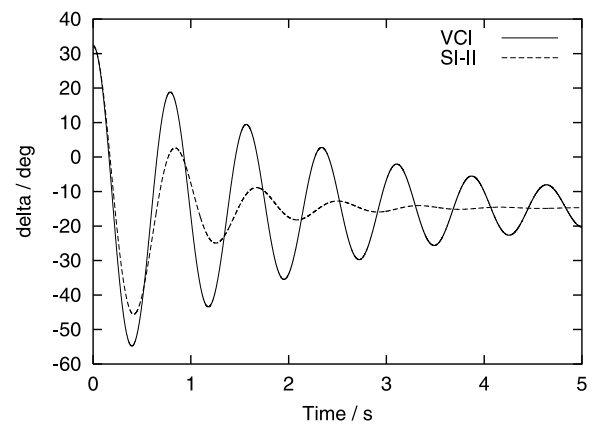

Fig. 11 Transient response caused by sudden drop of dc source voltage $E_{\mathrm{s}}$ from $60 \mathrm{~V}$ to $52 \mathrm{~V}$ with $L_{1}=L_{2}$.

is fixed at $1.2 \pi$. The disturbances are the same as discussed before.

As shown in Fig. 10, SI-II shows remarkably improved convergence over VCI. Depending on the design of PLL, SII had a worse response for the given disturbance. Therefore, the discussion on the effectiveness in comparison with other inverters is not significant.

In Fig. 9, SI-I does not show any advantages over VCI during a sudden drop of dc source voltage when the inverter is directly connected to the network. On the other hand, in Fig. 11, SI-II shows a remarkable improvement of transient behavior when indirectly connected.

Based on these numerical results, we can estimate that SIs can improve the transient response of the VCI under disturbances caused by distributed power source. In the next section, we confirm these characteristics by analyzing their transfer relation and poles.

\subsection{Relationships between Variations of Angles}

In this section, the transient characteristics of the linkage inverters are discussed. The relationships between variations of angles are easily obtained by the appropriate linearization of differential equations around an equilibrium point. The linearized relationships can be depicted by the polynomial function of $s$ and variables through Laplace transformation. They do not exist in the same state space depending on the system structure. Here the condition $L_{1}=L_{2}$ is set for simplicity.

As for VCI based on Eq. (4), the transfer relation from the initial variation $\delta^{\prime}(0)$ to $\delta^{\prime}$,

$$
\delta^{\prime}=\frac{s+\tau}{s^{2}+\tau s+A} \cdot \delta^{\prime}(0)
$$

is obtained. For SI-I based on Eq. (7), the transfer relation from the initial variations $\delta_{\mathrm{avr}}^{\prime}(0)$ and $\delta_{\mathrm{pll}}^{\prime}(0)$ to $\delta^{\prime}$,

$$
\delta^{\prime}=\frac{\delta^{\prime}(0) s^{2}+\left\{K_{\mathrm{p}} \delta_{\mathrm{avr}}^{\prime}(0)+\tau \delta^{\prime}(0)\right\} s+\tau K_{\mathrm{p}} \delta_{\mathrm{avr}}^{\prime}(0)}{s^{3}+\left(\frac{K_{\mathrm{p}}}{2}+\tau\right) s^{2}+\left(\frac{\tau K_{\mathrm{p}}}{2}+A\right) s+A K_{\mathrm{p}}}
$$

is obtained. Finally, for SI-II, Eq. (8) gives the transfer relation from the initial variations $\delta_{\mathrm{avr}}^{\prime}(0)$ and $\delta_{\mathrm{pll}}^{\prime}(0)$ to $\delta^{\prime}$ (Eq. (21)).

Here ( $)$ ' implies the variation of each value from a corresponding equilibrium point. $\mathrm{A}$ is defined by the phase of equilibrium point $\bar{\delta}$,

$$
A=\frac{K}{C} \cdot \frac{E}{2 X} \cdot \frac{1}{4+\alpha^{2}}(2 \cos \bar{\delta}-\alpha \sin \bar{\delta}) .
$$

If we fix the parameters as shown in Table 1 and $E_{\mathrm{OPT}}=50 \mathrm{~V}$, then Eq. (19) has poles at $-0.4167 \pm j 6.9090$ for setting $L_{1}=L_{2}=60 \mathrm{mH}$. Therefore, this ensures the transient behavior in the neighborhood of the equilibrium point.

The SI-I can adjust its stability by changing the gain $K_{\mathrm{p}}$ according to the disturbance. However, as mentioned earlier, the method is not designed for indirect connection to the power source. Figure 12 shows the locus of the real part of complex poles depending on the increase of $K_{\mathrm{p}}$. This result shows that SI-I has no stabilization at $K_{\mathrm{p}}>1.7$. This result does not conflict with the design concept of SI-II.

On the other hand, the complex conjugate poles of SIII show the locus of the real parts, as shown in Fig. 13. This ensures the stability of response under external disturbances. The locus has a minimal point, implying that the transient behavior can be optimized by tuning the gain $K_{\mathrm{p}}$.

This section shows that the transfer relation can explain the simulated results in Sect. 3.2. Moreover, it illustrates the advantages of SI-II in its optimal gain setting.

$$
\delta^{\prime}=\frac{\left(\delta_{\mathrm{avr}}^{\prime}(0)+\delta_{\mathrm{pll}}^{\prime}(0)\right) s^{2}+\left\{\left(\tau_{\mathrm{s}}+K_{\mathrm{p}}\right) \delta_{\mathrm{avr}}^{\prime}(0)+\tau_{\mathrm{s}} \delta_{\mathrm{pll}}^{\prime}(0)\right\} s+\tau_{\mathrm{s}} K_{\mathrm{p}} \delta_{\mathrm{avr}}^{\prime}(0)}{s^{3}+\left(\frac{3 K_{\mathrm{p}}}{2}+\tau_{\mathrm{s}}\right) s^{2}+\left(\frac{3 \tau_{\mathrm{s}} K_{\mathrm{p}}}{2}+A\right) s+A K_{\mathrm{p}}}
$$




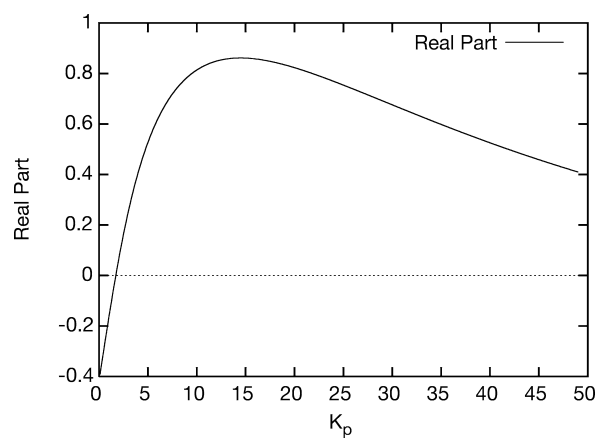

Fig. 12 Locus of real part of poles by SI-I.

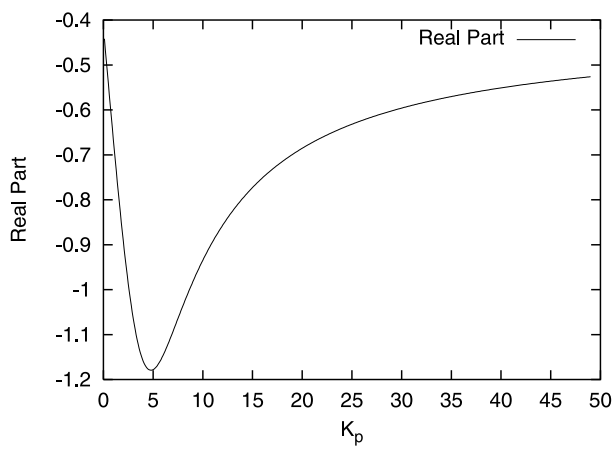

Fig. 13 Locus of real part of poles by SI-II.

\section{Experimental Examinations}

This section examines the characteristics of VCI and the SIs experimentally. The experiments are performed at the parameters set in Table 2 for the two disturbances we defined in the numerical simulations. The adopted parameters seem to be far from practical use of inverters. In this paper, however, the principles of the proposed control method for synchronous inverter are theoretically and numerically confirmed. Therefore, we adopted a micro experimental setup without loosing generality of system dynamics.

The characteristics of directly connected linkage inverters are examined in the case I. Figure 14 shows the transient responses during a sudden phase shift of ac voltage. SI-I shows an advantage over VCI in its transient decay. The result qualitatively coincides with the numerical simulation.

Figure 15 depicts the dependency of transient behavior on the capacitance. As expected through the simulation, the capacitance plays a role in inertia of dynamics in the linkage inverter system. Figure 16 experimentally shows that SI-I has no effect on the transient response when there is a sudden drop of dc source voltage as seen in Fig. 9.

In the former discussions, we mentioned that SI-I has no advantage in the connection to the power system though $L_{1}$ and $L_{2}$ because of its design. On the contrary, Fig. 17 shows that there is an effect for damping amplitude at the beginning of response better than VCI. However, this result is not ensured for other parameters. Moreover, the oscillation continues for a long time. On the other hand, Fig. 18
Table 2 Experimental setup.

\begin{tabular}{|c|c|c|c|c|c|}
\hline Case & $L_{1}$ & $L_{2}$ & VCI & SI-I & SI-II \\
\hline I & $60 \mathrm{mH}$ & 0 & Ref. [13] & $\begin{array}{c}\tau_{1}+\tau_{2}=5.4 \\
\tau_{2}=0.02 \\
K_{p}=8250\end{array}$ & \\
\hline II & $60 \mathrm{mH}$ & $60 \mathrm{mH}$ & Ref. [13] & $\begin{array}{c}\tau_{1}+\tau_{2}=5.4 \\
\tau_{2}=0.02 \\
K_{p}=8250\end{array}$ & $\begin{array}{c}\tau_{1}+\tau_{2}=1.5 \\
\tau_{2}=0.15 \\
K_{p}=82.5\end{array}$ \\
\hline
\end{tabular}

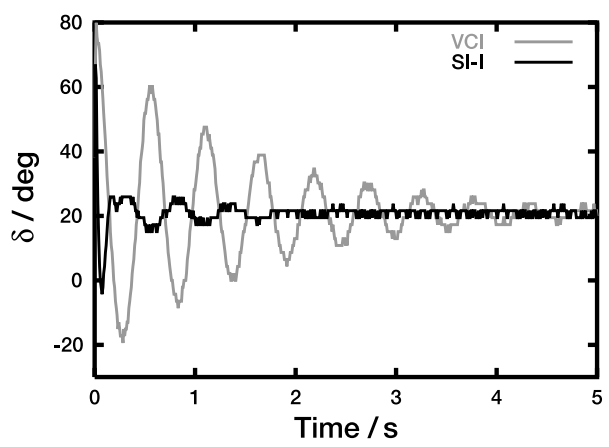

Fig. 14 Experimental results for sudden phase shift of ac voltage of power system from 0 to $0.3 \pi(\mathrm{Lag})$ with $L_{2}=0$.

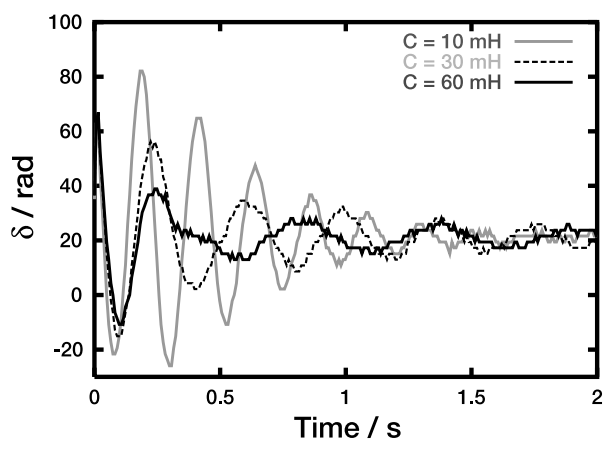

Fig. 15 Experimental results for dependency of transient response of SII on capacitance $C$ while keeping $L_{2}=0$.

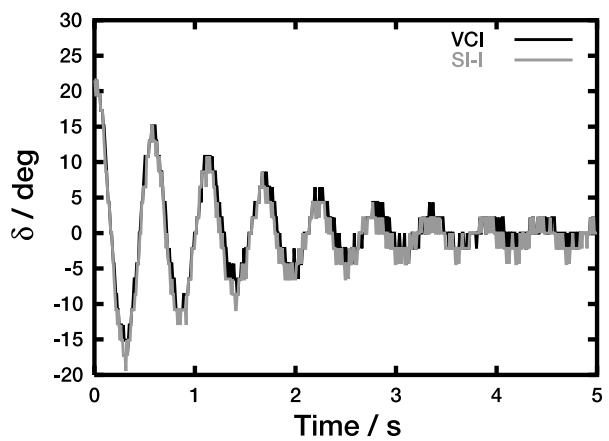

Fig. 16 Experimental results for sudden voltage drop of dc source voltage $E_{\mathrm{s}}$ from $60 \mathrm{~V}$ to $52 \mathrm{~V}$ with $L_{2}=0$.

shows that SI-I has no advantage when there is a sudden drop in dc source voltage.

Figures 19 and 20 confirm the effectiveness of SI-II. For both types of disturbances, SI-II has improved transient response. Therefore, it is experimentally confirmed that SI- 


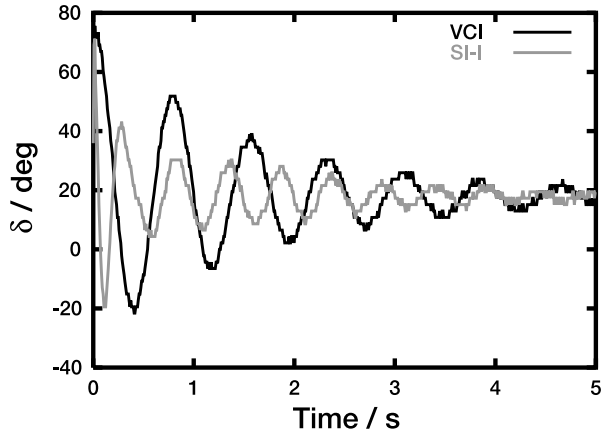

Fig. 17 Experimental results for sudden phase shift of ac voltage of power system from 0 to $0.3 \pi$ (Lag) with $L_{2}=L_{1}$ to see the effect of SI-I.

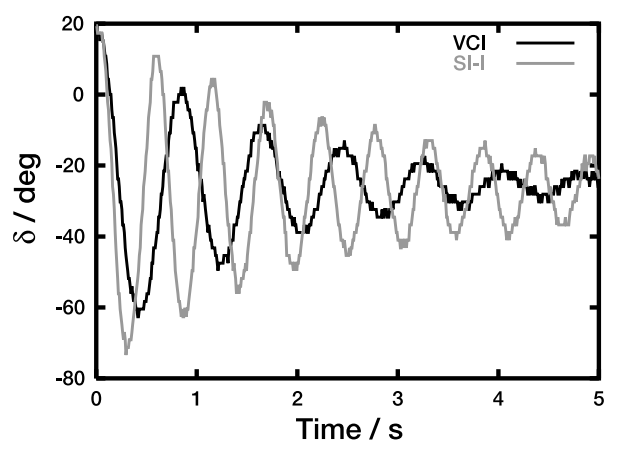

Fig. 18 Experimental results for sudden voltage drop of dc source voltage $E_{\mathrm{s}}$ from $60 \mathrm{~V}$ to $52 \mathrm{~V}$ with $L_{2}=L_{1}$ to see the effect of SI-I.

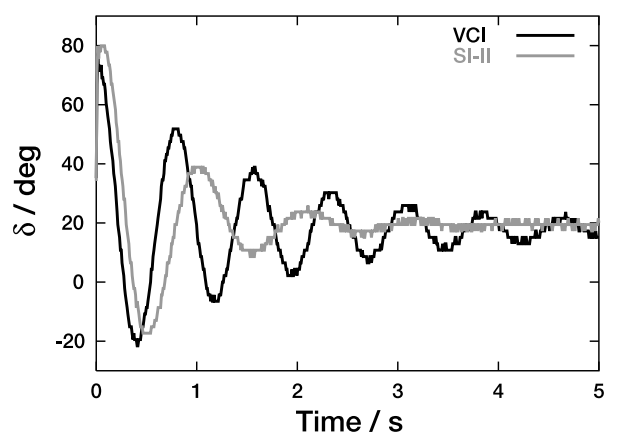

Fig. 19 Experimental results for sudden phase shift of ac voltage of power system from 0 to $0.3 \pi(\mathrm{Lag})$ with $L_{2}=L_{1}$ to see the effect of SI-II.

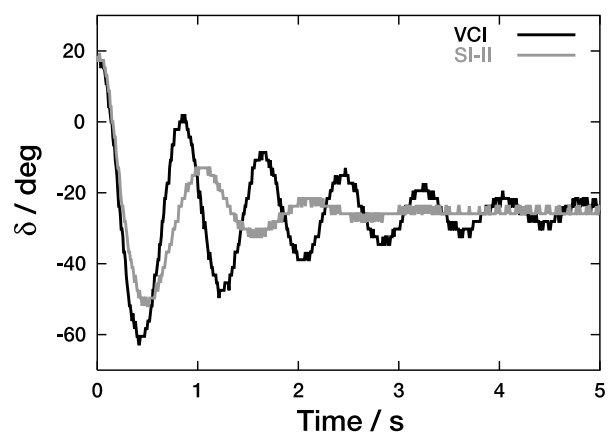

Fig. 20 Experimental results for sudden voltage drop of dc source voltage $E_{\mathrm{s}}$ from $60 \mathrm{~V}$ to $52 \mathrm{~V}$ with $L_{2}=L_{1}$ to see the effect of SI-II.
II has strong self-synchronizing characteristics to work with power delivery system under disturbances.

\section{Transient Dynamics of Linkage Inverter and En- trainment}

The linkage inverter systems discussed in this paper show self-synchronizing characteristics based on their frequency and phase entrainment characteristics. We can estimate the improvement of the characteristics through the basin; the domain of attraction in initial values space. The estimated trajectory from the initial value shows the pull-in characteristics after the onset of control.

In the following simulations, the parameters are set as follows: $C=60 \mathrm{mF}, K=2.4 \pi, E_{\mathrm{s}}=60 \mathrm{~V}, E_{\mathrm{OPT}}=50 \mathrm{~V}$, $R_{\mathrm{S}}=20 \Omega, L_{1}=60 \mathrm{mH}$, and $E_{\mathrm{AC}}=50 \mathrm{~V}$.

\subsection{Feature of VCI}

\subsubsection{Basin Portrait}

VCI has the domain of attraction shown in Fig. 21. On the $(\delta, \omega)$-plane, the solution obtained from every initial value in the hatched region (domain) converges to the equilibrium point (black circle) at the center of the domain. The domain has boundaries consisting of the stable manifolds which converges to a saddle, marked by a black, downword pointing triangle in the figure. The feature is similar to synchronous generators. The domain decreases as the dc voltage $E_{\mathrm{s}}$ increases.

\subsubsection{Pull-in Characteristics}

From the viewpoint of nonlinear dynamics, VCI inherently has a self-synchronizing parameter area (shown in Fig. 22). However, the transient characteristic is not sufficient. The damping is low to obtain quick convergence.

The trajectory diverges from the equilibrium point because of sudden decrease of $E_{\mathrm{s}}$ from $60 \mathrm{~V}$ to $30 \mathrm{~V}$. The recovery time of $E_{\mathrm{s}}$ to $60 \mathrm{~V}$ determines the stability of operation. If the recovery time is slow, then the state cannot be pulled to the equilibrium point. The critical point exists on the boundary of the basin.

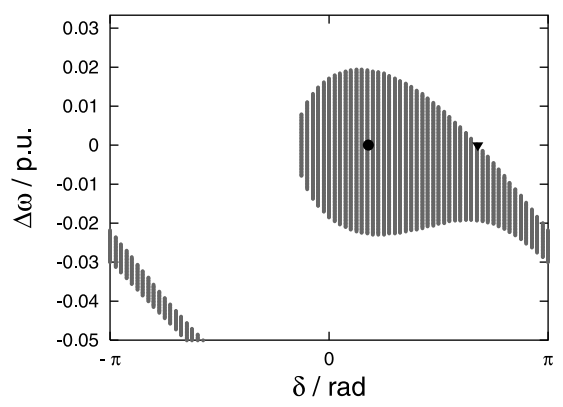

Fig. 21 Basin obtained for VCI. 


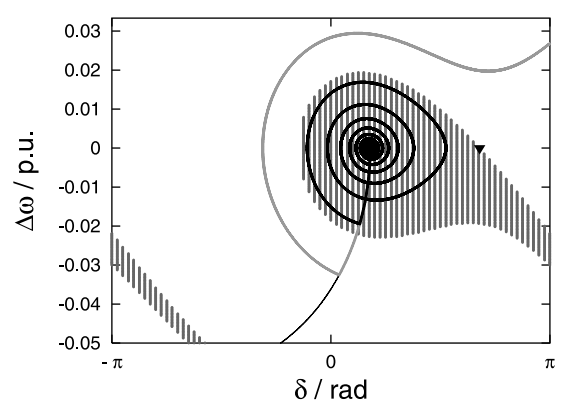

Fig. 22 Pull-in characteristics of VCI. The trajectry shows the convergence from the initial value inside domain and the divergence outside.

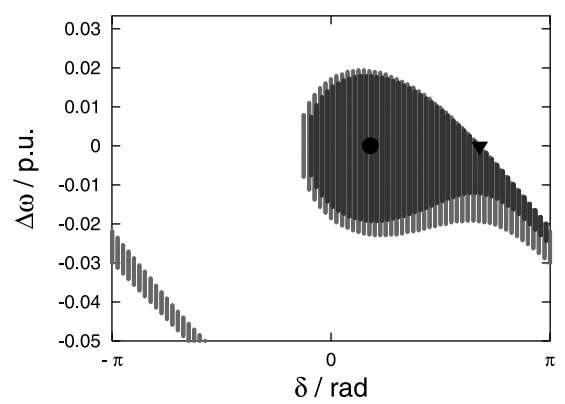

Fig. 23 Basin obtained for SI-I (black) compared with VCI (dark gray).

\subsection{Feature of SIs}

SIs have higher dimensions than VCI. Therefore, it is difficult to compare their characteristics in the same $(\delta, \omega)$-plane. Here we discuss the characteristics through projections on the plane without respect to changes of the control variables.

The SI-I has an additional PLL to acheive the phase synchronization under the frequency synchronization. The PLL substantially synchronizes its phase to the reference signal. As shown in the previous section, VCI has a selfsynchronizing characteristic similar to that of synchronous generators, which operates according to the power flow. In fixed reference frequency, SI-I has no effective characteristics. In addition, the basin of the equilibrium point is narrowed down for SI-I as shown in Fig. 23. The tracking of the frequency of the power delivery system makes the system unstable under the transient state. However, we have shown in the former discussion that the system has better damping characteristics.

The SI-II shows remarkable enlargement of the basin under control, as shown in Fig. 24, particularly in the region of negative high angular frequency. This implies the SI-II can stabilize large frequency fluctuations. The enlargement into the low frequency area has a remarkable effect in the application of the distributed power sources.

\section{Conclusions}

This paper proposed synchronous inverters for distributed power sources based on voltage controlled inverters with

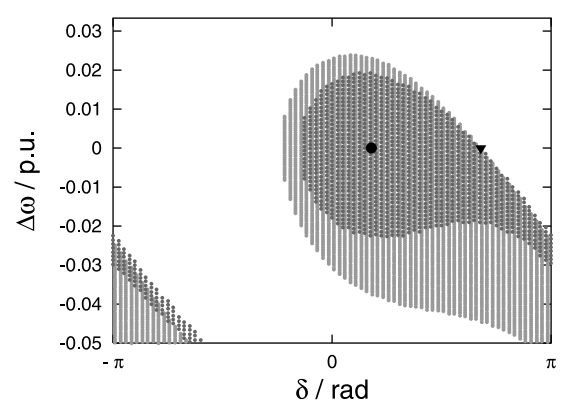

Fig. 24 Basin obtained for SI-II (light gray) compared with VCI (dark gray).

a modified PLL. The characteristics are discussed numerically, analytically, and experimentally. This approach is strongly related to the study which investigates the relationship between synchronization and power transfer by Susuki et al. [14].

Conventionally, the linkage inverters for distributed power sources have been applied in the low functional level from the viewpoint of control. That is, they have been used only as converters between dc and ac power in the algebraic restriction. However, power systems increasingly require all system equipment to synchronize for optimum electric power transfer. As a result, the conventional inverters occasionally lose the synchronization under disturbances, due to designs that support only one-directional synchronization control methods. The inverters discussed in this paper can maintain frequency and phase synchronization similar to the synchronous generators.

The idea of the self-synchronizing inverter was proposed by Harada et al. as a voltage controlled inverter. We showed that the phase-synchronization characteristics can be improved by the addition PLL with keeping the steady state characteristics for maximum power point tracking. The improved inverters showed good entrainment characteristics during transients, even during a large initial disturbance. This shows that the distributed power sources have the potential to contribute the stable operation of power distribution system by integrating numbers of the individual systems. We called these inverters synchronous inverters.

The authors are to see the application of synchronous inverters and the development of novel electric power networks. Highly connected synchronous systems may show the phenomena of nonlinear dynamics described by small world physics [15]. We have a potential to regulate these systems through functional inverters distributed throughout the networks.

\section{Acknowledgement}

The authors acknowledge Prof. Yoshihiko Susuki for his fruitful comments and discussion on synchronization. This work was supported by Iwatani Naoji Foundation's Reserch Grant and the Japan Society for the Promotion of Science (JSPS) the 21st Century COE Program (Grant No. 14213201). 


\section{References}

[1] A.T. Winfree, The Geometry of Biological Time, 2nd ed., Springer, 2000.

[2] S. Strogatz, Sync, Hyperion Books, 2003.

[3] Y. Kuramoto, Chemical Oscillations, Waves, and Turbulence, Dover, 1984.

[4] M.P. Kennedy and M.J. Ogozalek, eds., Special Issue on Chaos Synchronization and Control: Theory and Applications, IEEE Trans. CAS-I, vol.44, no.10, 1997.

[5] E.W. Kimbark, Power System Stability, IEEE Press, 1995.

[6] C. Gellings, "Transforming the electric infrastructure," Physics Today, vol.57, no.12, pp.45-51, 2004.

[7] P. Fairley, "The unruly power grid," IEEE Spectr., vol.41, no.8, pp.22-27, Aug. 2004.

[8] Y. Li, D.M. Vilathgamuwa, and P.C. Loh, "Design, analysis, and real-time testing of a controller for multibus microgrid system," IEEE Trans. Power Electron., vol.19, no.5, pp.1195-1204, 2004.

[9] D.G. Infield, P. Onions, A.D. Simmons, and G.A. Smith, "Power quality from multiple grid-connected single-phase inverters," IEEE Trans. Power Deliv., vol.19, no.4, pp.1983-1989, 2004.

[10] J. Holtz, "Pulse width modulation for electronic power conversion," in Power Electronics and Variable Frequency Drives, ed. B.K. Bose, Chap. 4, IEEE Press, 1997.

[11] S.A. Oliveira da Silva, P.F. Donoso-Garcia, P.C. Cortizo, and P.F. Seixas, "A three-phase line-interactive UPS system implementation with series-parallel active power-line conditioning capabilities," IEEE Trans. Ind. Appl., vol.38, no.6, pp.1581-1590, 2002.

[12] S.J. Lee, J.K. Kang, and S.K. Sul, "A new phase detecting method for power conversion systems considering distorted conditions in power system," Proc. IAS Annual Meeting, pp.2167-2172, 1999.

[13] K. Harada and K. Murata, "Interface circuit between solarcell and commercial AC bus," IEICE Trans. Electron. (Japanese Edition), vol.J69-C, no.11, pp.1458-1464, Nov. 1986.

[14] Y. Susuki, Y. Yokoi, and T. Hikihara, "Energy-based analysis of frequency entrainment described by van der Pol and PLL equations," CHAOS, vol.17, 023108, 2007.

[15] D.J. Watts, Small Worlds, Princeton Univ. Press, 1999.

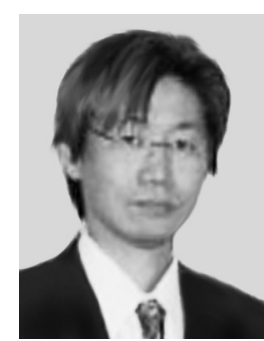

Takashi Hikihara received Ph.D. degree from Kyoto University, in 1990. Since 1997 he has been with Department of Electrical Engineering at Kyoto University, where he is currently a Professor. His research interests are including nonlinear science, analysis of nonlinear system, nano-mechanical systems, and power engineering. He is currently an associate editor of Journal of Circuits, Systems, and Computers. $\mathrm{He}$ is a regular member of the IEEE, IEE, APS, SIAM, and IEE, Japan.

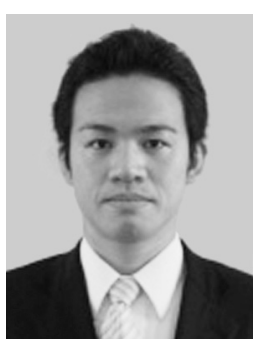

Tadashi Sawada received the B.E. and M.E. degrees from Kyoto University in 2003 and 2005 respectively. In Kyoto University, he has been engaged in the project of synchronizing inverter for distributed power sources. $\mathrm{He}$ joined to JR Central in 2005.

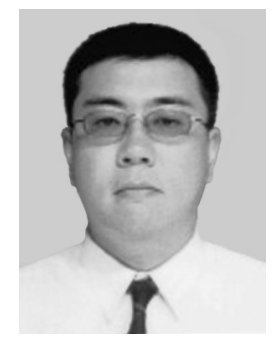

Tsuyoshi Funaki received the B.E. M.E., and $\mathrm{Ph} . \mathrm{D}$. degrees from Osaka University. $\mathrm{He}$ joined Osaka University as research associate in 1994, assistant professor in 2001. He joined Kyoto University as associate professor in 2002 $\mathrm{He}$ is a member of IEE Japan, ISCIE, JSER, SAEJ, IEEE, IET. 